\title{
TWISTING COCHAINS AND DUALITY BETWEEN MINIMAL ALGEBRAS AND MINIMAL LIE ALGEBRAS
}

BY

RICHARD M. HAIN

\begin{abstract}
An algebraic duality theory is developed between 1-connected minimal cochain algebras of finite type and connected minimal chain Lie algebras of finite type by means of twisting cochains. The duality theory gives a concrete relationship between Sullivan's minimal models, Chen's power series connections and the various Lie algebra models of a 1-connected topological space defined by Quillen, Allday, Baues-Lemaire and Neisendorfer. It can be used to compute the Lie algebra model of a space from the algebra model of the space and vice versa.
\end{abstract}

1. Introduction. Baues and Lemaire [2] have developed an algebraic duality theory between 1-connected minimal cochain algebras of finite type and connected minimal chain Lie algebras of finite type. The duality theory gives a one-to-one correspondence between isomorphism classes of 1-connected minimal cochain algebras of finite type and isomorphism classes of connected minimal chain Lie algebras of finite type. If the cochain algebra $\Re$ corresponds to the chain Lie algebra $L$ under the duality theory, then there are natural, nonsingular pairings

$$
Q \Re \otimes s H_{*}(L) \rightarrow \mathbf{Q}
$$

and

$$
I H^{*}(\Re) \otimes s Q L \rightarrow \mathbf{Q}
$$

where $Q \Re$ denotes the space of indecomposables of $\Re, Q L$ denotes $L /[L, L], s$ denotes the suspension operator and $I H^{*}(\mathscr{N})=\bigoplus_{p>0} H^{p}(\mathscr{N})$. The duality theory reflects the adjointness of Quillen's $\mathcal{L}_{-}$and $\mathrm{C}_{\text {-constructions. }}$

For a 1-connected space $K$ with finite Betti numbers, Sullivan's minimal model $\mathfrak{\pi}_{K}$ of $K$ is a 1-connected minimal cochain algebra of finite type which has the properties

$$
Q \Re_{K} \approx \operatorname{Hom}_{\mathbf{Z}}\left(\pi_{*}(K), \mathbf{Q}\right) \text { and } H^{*}\left(\Re_{K}\right) \approx H^{*}(K ; \mathbf{Q})
$$

(see $[6,15])$. The primitive elements $L_{K}$ of Chen's algebra model of $K($ see $[5,18])$ is a connected minimal chain Lie algebra of finite type which has the properties

$$
Q \Re_{K} \approx \operatorname{Hom}_{\mathbf{z}}\left(\pi_{*}(K), \mathbf{Q}\right) \text { and } H^{*}\left(\Re_{K}\right) \approx H^{*}(K ; \mathbf{Q})
$$

Received by the editors February 2, 1981 and, in revised form, May 10, 1982; presented to the Society, April 11, 1980.

1980 Mathematics Subject Classification. Primary 55P62, 55U30.

Key words and phrases. Minimal algebra, minimal Lie algebra, formal homology connection. 
(see $[6,15]$ ). The Lie algebra model of $K$ obtained by using K. T. Chen's method of power series connections (see $[\mathbf{5}, \mathbf{1 8}]$ ) is a minimal chain Lie algebra which has the properties

$$
s H_{*}\left(L_{K}\right) \approx \pi_{*}(K) \otimes_{\mathbf{Z}} \mathbf{Q} \text { and } s Q L_{K} \approx \tilde{H}_{*}(K ; \mathbf{Q}) .
$$

(Similar Lie algebra models have been studied by Quillen [14], Allday [1], Baues and Lamaire [2] and Neisendorfer [13].) Under the duality theory, $\mathfrak{T}_{K}$ and $L_{K}$ correspond, and the pairings (1.1) and (1.2) correspond to the natural pairings

$$
\operatorname{Hom}_{\mathbf{z}}\left(\pi_{*}(K), \mathbf{Q}\right) \otimes\left(\pi_{*}(K) \otimes_{\mathbf{z}} \mathbf{Q}\right) \rightarrow \mathbf{Q}
$$

and

$$
\tilde{H}^{*}(K ; \mathbf{Q}) \otimes \tilde{H}_{*}(K ; \mathbf{Q}) \rightarrow \mathbf{Q} .
$$

A direct consequence of the duality theory is that Chen's model of a 1-connected space $K$ with finite Betti numbers is isomorphic to the minimal model of the $\mathcal{L}$-construction $\mathcal{L}\left(\mathfrak{R}_{K}^{*}\right)$ on the dual of Sullivan's model $\mathfrak{K}_{K}$ of $K$.

Given a 1-connected minimal cochain algebra $\mathfrak{T}$ of finite type, the twisting cochain $\omega$ and the corresponding Lie algebra $L$ can be computed inductively from

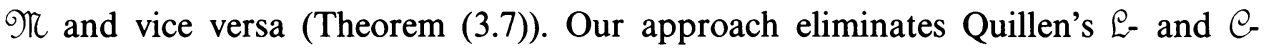
constructions from the duality theory and, in the author's opinion, makes the computation of the correspondence and the pairings (1.1) and (1.2) simpler and more tractable than when using the Baues-Lemaire approach.

Independently, Tanré $[19,20]$ has obtained results that relate Chen's power series connections and Chen's Lie algebra model of a space to other algebraic models of the space. Specifically, he has shown that there is a natural one-to-one correspondence between power series connections on a cochain algebra $Q$ and Felix-HalperinStasheff (F.H.S.) models $(\Lambda Z, d) \rightarrow \mathscr{Q}$ of $\mathscr{Q}$ (see [17]). In the case when $\mathscr{Q}$ is the de Rham complex of a wedge of spheres, the F.H.S. model of $Q$ equals the Sullivan model of $\mathcal{Q}$ and Tanré's result and ours coincide. It also follows from Tanré's result that Chen's algebra model of a space is isomorphic to the Adams-Hilton model of the space. Tanré's methods are similar to ours.

Let $K$ be a 1-connected rational space with finite Betti numbers. In [18], it is proved that elementary extensions of Chen's Lie algebra model of $K$ correspond to cofibrations $K \rightarrow K \cup_{f} e_{(0)}^{n}$ where $e_{(0)}^{n}$ is a rational cell of dimension $n$ (that is, the cone over a rational $(n-1)$ sphere $\left.S_{(0)}^{n-1}\right)$ and where $f_{*}: H_{n-1}\left(S_{(0)}^{n-1}\right) \rightarrow H_{n-1}(K)$ is trivial. Thus Chen's Lie algebra model of $K$ determines a rational homology decomposition of $K$. Since Sullivan's model of $K$ determines a Postnikov tower of $K$, the duality theory gives a method of computing the rational cell structure of $K$ from a Postnikov tower of $K$ and vice versa. This result is related to the Baues-Lemaire conjecture [2] which asserts that the minimal model of Quillen's Lie algebra model of

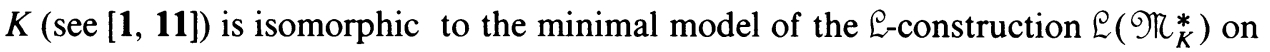
the dual of Sullivan's model $\Re_{K}$ of $K$.

The results in this paper are excerpted from the author's Ph.D. thesis [8] which was supervised by Professor Kuo-Tsai Chen to whom the author wishes to express 
his sincere gratitude for guidance and encouragement given during the preparation of the thesis.

The first four sections of this paper are elementary and self-contained. $\S 2$ is a list of notation and conventions used in this paper. In $\$ 3$ connections on cochain algebras and chain Lie algebras are defined and their existence is proved. In $\S 4$ we state the main theorem of this paper and discuss its topological implications. \$5 contains a proof of the main theorem.

2. Notation and conventions. A graded vector space $V$ is a sequence $\left\{V_{p}\right\}$ of $\mathbf{Q}$-vector spaces where $p$ runs through the integers $\mathbf{Z}$. The degree of an element $v$ of $V$ will be denoted by deg $v$. The graded vector space is said to be of finite type if each $V_{p}$ has finite dimension. Let $r \in \mathbf{Z}$. We say that $V$ is $r$-connected if $V_{p}=0$ whenever $p \leqslant r$. The $r$-fold suspension $s^{r} V$ of $V$ is the graded vector space defined by $\left(s^{r} V\right)_{p}=V_{p-r}$. The ground field $\mathbf{Q}$ will be considered as a graded vector space concentrated in degree zero.

For a differential graded (d.g.) vector space $V$ and a cycle $v$ in $V$, we shall denote the homology class of $v$ by $\{v\}$.

A cochain algebra is a differential graded algebra whose differential has degree +1 . That is, $\mathbb{Q}$ is a cochain algebra with differential $d$ and if $a \in \mathbb{Q}^{p}$ and $b \in \mathbb{Q}^{q}$, then

$$
\begin{gathered}
a \wedge b=(-1)^{p q} b \wedge a, \\
d(a \wedge b)=(d a) \wedge b+(-1)^{p} a \wedge(d b)
\end{gathered}
$$

and, in addition, there is a d.g. algebra map $\varepsilon: \mathbb{Q} \rightarrow \mathbf{Q}$ of degree 0 . The kernel of $\varepsilon$ is called the augmentation ideal of $\mathbb{Q}$ and will be denoted by $I Q$. The $r$ th power of $I \mathbb{Q}$ will be denoted by $I^{r} Q$. The space of indecomposables of $Q$ is the d.g. vector space $I Q / I^{2} Q$ and will be denoted by $Q Q$. Let $r$ be a nonnegative integer. We say that $Q$ is $r$-connected if the graded vector space $I Q$ is $r$-connected.

We shall denote the free graded commutative algebra generated by the graded vector space $V$ by $\Lambda(V)$. That is, $\Lambda(V)$ is the tensor product of the polynomial algebra generated by the elments of $V$ of even degree with the exterior algebra generated by the elements of $V$ of odd degree. The free graded commutative algebra generated by the graded set $\left\{x_{1}, x_{2}, \ldots\right\}$ will be denoted by $\Lambda\left(x_{1}, x_{2}, \ldots\right)$.

A chain Lie algebra is a graded Lie algebra whose differential has degree -1 . That is, if $L$ is a chain Lie algebra with differential $\partial$ and if $X \in L_{p}, Y \in L_{q}$ and $Z \in L_{r}$, then

$$
\begin{gathered}
{[X, Y]+(-1)^{p q}[Y, X]=0,} \\
(-1)^{p r}[X[Y, Z]]+(-1)^{q p}[Y[Z, X]]+(-1)^{r q}[Z[X, Y]]=0, \\
\partial[X, Y]=[\partial X, Y]+(-1)^{p}[X, \partial Y] .
\end{gathered}
$$

The subspace $[L, L]$ of $L$ will be denoted by $I^{2} L$. For $r>2$ set $I^{r} L=\left[L, I^{r-1} L\right]$. The space of indecomposables of $L$ is the d.g. vector space $L / I^{2} L$ and will be denoted by $Q L$. Let $r$ be a nonnegative integer. A chain Lie algebra is said to be $r$-connected if it is $r$-connected as a graded vector space. 
The free graded Lie algebra generated by the graded vector space $V$ will be denoted by $\mathbf{L}(V)$. The free graded Lie algebra generated by the graded set $\left\{X_{1}, X_{2}, \ldots\right\}$ will be denoted by $\mathbf{L}\left(X_{1}, X_{2}, \ldots\right)$.

3. Connections. Twisting cochains are the backbone of the duality theory. As they are viewed in this paper, they provide a concrete means for computing the minimal chain Lie algebra $L$ that corresponds to a given minimal cochain algebra $\Re$, and vice versa, as well as the associated pairings

$$
Q \Re \otimes s H_{*}(L) \rightarrow \mathbf{Q}, \quad I H^{*}(\Re) \otimes s Q L \rightarrow \mathbf{Q} .
$$

The twisting cochains that arise in the duality theory are called connection forms, the terminology used by Chen in the analogous topological situation $[4,5]$.

Let $V, W$ be graded vector spaces. Denote by $V \hat{\otimes} W$ the graded vector space $\prod_{n=0}^{\infty} \bigoplus_{p+q=n} V_{p} \otimes W_{q}$. A typical element of $V \hat{\otimes} W$ being the infinite sum $\sum_{j=0}^{\infty} v_{j}$ $\otimes w_{j}$ where, for each $n$, there is only a finite number of terms $v_{j} \otimes w_{j}$ of degree $n$.

For each cochain algebra $\mathbb{Q}$, define a linear automorphism $J: \mathbb{Q} \rightarrow \mathbb{Q}$ by $J a=$ $(-1)^{\operatorname{deg} a} a$ for each $a \in \mathbb{Q}$.

Let $Q$ be a cochain algebra with differential $d$ and $L$ a chain Lie algebra with differential $\partial$. We can extend the actions of $d, \partial, J$ to $Q \hat{\otimes} L$ by setting $d\left(\sum a_{j} \otimes X_{j}\right)$ $=\Sigma\left(d a_{j}\right) \otimes X_{j}, \partial\left(\Sigma a_{j} \otimes X_{j}\right)=\Sigma a_{j} \otimes\left(\partial X_{j}\right), J\left(\Sigma a_{j} \otimes X_{j}\right)=\Sigma\left(J a_{j}\right) \otimes X_{j}$ where $a_{j}$ $\in \mathbb{Q}$ and $X_{j} \in L$. Define a bracket [,] in $Q \hat{\otimes} L$ by $\left[\Sigma a_{i} \otimes X_{i}, \Sigma b_{j} \otimes Y_{j}\right]=$ $\Sigma\left(a_{i} \wedge b_{j}\right) \otimes\left[X_{i}, Y_{j}\right]$ where $a_{i}, b_{j} \in \mathbb{Q}$ and $X_{i}, Y_{j} \in L$. Usually we will omit the tensor product sign and write $\sum a_{i} \otimes X_{i}$ as $\sum a_{i} X_{i}$.

(3.1) Definition. Let $L$ be a chain Lie algebra (with differential $\partial$ ) and $\mathbb{Q}$ a cochain algebra (with differential $d$ ). A twisting cochain in $Q \hat{\otimes} L$ is an element $\omega$ of $\prod_{p=0}^{\infty} \mathbb{Q}^{p+1} \otimes L_{p}$ satisfying the twisting cochain condition

$$
\partial \omega+d \omega-\frac{1}{2}[J \omega, \omega]=0 .
$$

If $Q$ is of finite type, then $\operatorname{Hom}(Q, Q)$ is a d.g. coalgebra and the map $\tau_{\omega}$ : $\operatorname{Hom}(\mathbb{Q}, \mathbf{Q}) \rightarrow L$ defined by $\tau_{\omega}(\varphi)=\sum \varphi\left(a_{i}\right) A_{i}$, where $\omega=\sum a_{i} A_{i}$ and $\varphi \in$ $\operatorname{Hom}(Q, Q)$, is a twisting cochain in the sense of Brown [3]. For our purposes there are two advantages in representing twisting cochains as elements of $\mathcal{Q} \hat{\otimes} L$ rather than as functions. The first is that, in our context, it is more natural to deal with $\mathscr{Q}$, usually a minimal cochain algebra of a de Rham complex, than its dual. Second, twisting cochains take on a very explicit form when written as elements of $\mathcal{Q} \hat{\otimes} L$. This is an advantage, especially in computations.

The association between a minimal cochain algebra and a minimal chain Lie algebra is made by means of a connection.

(3.2) Definitions. (a) Let $\mathscr{Q}$ be a cochain algebra with 1-connected homology and finite Betti numbers. A connection on $Q$ is a free chain Lie algebra $L$ and twisting cochain $\omega$ in $Q \hat{\otimes} L$ such that if $\left(X_{i}\right)$ is a set that freely generates $L$ and if

$$
\omega \equiv \sum w_{i} X_{i} \bmod \Theta \hat{\otimes} I^{2} L,
$$

then each $w_{i}$ is closed in $\mathcal{Q}$ and their homology classes $\left(\left\{w_{i}\right\}\right)$ form a basis of $I H^{*}(a)$.

We shall denote a connection on $Q$ by $(\omega, L)$ and shall call $\omega$ the connection form of the connection $(\omega, L)$. 
(b) Let $L$ be a chain Lie algebra with connected homology and finite Betti numbers. A connection on $L$ is a free cochain algebra $\Re$ and a twisting cochain $\omega$ in $\Re \hat{\otimes} L$ such that if $\left(x_{i}\right)$ is a set that freely generates $\Re$ and if

$$
\omega \equiv \sum x_{i} W_{i} \bmod I^{2} \mathfrak{N} \hat{\otimes} L,
$$

then each $W_{i}$ is closed in $L$ and their homology classes $\left(\left\{W_{i}\right\}\right)$ form a basis of $H_{*}(L)$.

We shall denote a connection on $L$ by $(\Re, \omega)$ and shall call $\omega$ the connection form of the connection $(\Re, \omega)$.

Note that since $\omega$ is a twisting cochain then $\operatorname{deg} w_{i}=1+\operatorname{deg} X_{i}$ and $\operatorname{deg} x_{i}=-1$ $+\operatorname{deg} W_{i}$.

(3.3) ExAmples. (a) If $\mathscr{Q}=\Lambda\left(a, b\right.$ : $\left.\operatorname{deg} a=2, \operatorname{deg} b=3, d a=0, d b=a^{2}\right)$, then a connection $(\omega, L)$ on $Q$ is given by

$$
L=\mathbf{L}(X: \operatorname{deg} X=1, \partial X=0), \quad \omega=a X+\frac{1}{2} b[X, X] .
$$

(b) If $L=\mathbf{L}(X, Y, Z: \operatorname{deg} X=\operatorname{deg} Y=2, \operatorname{deg} Z=5, \partial X=\partial Y=0, \partial Z=[X, Y])$, then a connection $(\Re, \omega)$ on $L$ is given by

$$
\Re=\Lambda(a, b: \operatorname{deg} a=\operatorname{deg} b=3, d a=d b=0), \quad \omega=a X+b Y+J a \wedge b Z .
$$

Observe that if $\mathscr{Q}$ is a 1-connected cochain algebra with finite Betti numbers and $(\omega, L)$ is a connection on $\mathbb{Q}$, then there is a nonsingular pairing

$$
I H^{*}(Q) \otimes s Q L \rightarrow \mathbf{Q}
$$

defined as follows: if $\omega \equiv \sum w_{i} X_{i} \bmod \Theta \hat{\otimes} I^{2} L$, then $\left\{w_{i}\right\} \otimes s X_{j} \mapsto \delta_{i j}$. Since the pairing is nonsingular, it follows that as a graded Lie algebra

$$
L \approx \mathbf{L}\left(s^{-1} \operatorname{Hom}\left(I H^{*}(\mathbb{Q}), \mathbf{Q}\right)\right) .
$$

In the dual situation, a connection $(\Re, \omega)$ on a connected chain Lie algebra $L$ defines a nonsingular pairing

$$
Q \Re \otimes s H_{*}(L) \rightarrow \mathbf{Q}
$$

Because the pairing is nonsingular, it follows that as a graded algebra $\Re \approx$ $\Lambda\left(s \operatorname{Hom}\left(H_{*}(L), \mathbf{Q}\right)\right)$.

To prove the existence of a connection on a cochain algebra $Q$, we have to define a suitable differential on $L=\mathbf{L}\left(s^{-1} \operatorname{Hom}\left(I H^{*}(Q), Q\right)\right)$ and a connection form in $Q \hat{\otimes} L$. Dually, to prove the existence of a connection on a chain Lie algebra $L$ we have to find a suitable differential on $\Re=\Lambda\left(\sin \left(H^{*}(L), \mathbf{Q}\right)\right)$ and a connection form in $\Re \hat{\otimes} L$. The following theorem asserts that connections always exist. Its proof is constructive. First we recall the definitions of minimal cochain algebras and minimal chain Lie algebras.

(3.6) Definitions. (a) A connected cochain algebra $\mathfrak{N}$ is said to be a minimal cochain algebra if:

(i) $\mathfrak{N}$ is a free graded commutative algebra,

(ii) its differential $d$ is decomposable; that is, $d(I \Re) \subseteq I^{2} \Re$,

(iii) there is a chain

$$
\mathbf{Q}=\mathfrak{K}_{0} \subseteq \mathfrak{K}_{1} \subseteq \mathfrak{K}_{2} \subseteq \cdots \subseteq \mathfrak{K}
$$

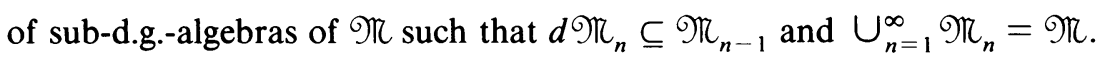


When $\Re$ is 1-connected (iii) follows from (ii).

(b) A chain Lie algebra $L$ is said to be a minimal chain Lie algebra if:

(i) $L_{p}=0$ whenever $p<0$,

(ii) $L$ is a free graded Lie algebra,

(iii) its differential $\partial$ is decomposable; that is, $\partial L \subseteq[L, L]$.

(3.7) THEOREM. (a) Every cochain algebra with 1-connected homology of finite type has a connection. The chain Lie algebra associated to such a cochain algebra by a connection is a connected minimal chain Lie algebra.

(b) Every chain Lie algebra with connected homology of finite type has a connection. The cochain algebra associated to such a chain Lie algebra by a connection is a 1-connected minimal cochain algebra.

Proof. We prove only (b). The proof of (a) is an algebraic analogue of Chen's proof of Theorem 1.3.1 in [4] and is dual to the proof of (b). Gugenheim has proved an assertion similar to (a) in [7].

Let $L$ be a chain Lie algebra with connected homology of finite type. Set $\mathfrak{N}=\Lambda\left(s \operatorname{Hom}\left(H_{*}(L), \mathbf{Q}\right)\right)$. Choose a basis $\left(x_{i}\right)_{i \in S}$ of $s \operatorname{Hom}\left(H_{*}(L), \mathbf{Q}\right)$. Choose closed elements $\left(W_{i}\right)_{i \in S}$ of $L$ such that their homology classes $\left(\left\{W_{i}\right\}\right)_{i \in S}$ form a basis of $H_{*}(L)$ dual to the basis $\left(s^{-1} x_{i}\right)$ of $\operatorname{Hom}\left(H_{*}(L), \mathbf{Q}\right)$. We have to find a decomposable differential $d$ on $\Re$ and a twisting cochain $\omega$ in $\Re \hat{\otimes} L$ such that

$$
\omega \equiv \sum x_{i} W_{i} \bmod I^{2} \mathscr{R} \hat{\otimes} L \quad \text { and } \partial \omega+d \omega-\frac{1}{2}[J \omega, \omega]=0
$$

For this we need the following lemma.

(3.8) Lemma. Let $n$ be a positive integer. If $d$ is a derivation of $\Re$ of degree 1 such that $d(I \Re) \subseteq I^{2} \Re$ and if $\omega$ is an element of $\Pi_{p=0}^{\infty} \Re^{p+1} \otimes L_{p}$ such that:

(a) $\omega \equiv \Sigma x_{i} W_{i} \bmod I^{2} \Re \hat{\otimes} L$,

(b) $\partial \omega+d \omega-\frac{1}{2}[J \omega, \omega] \equiv 0 \bmod I^{n} \Re \hat{\otimes} L$, then

(c) $\partial\left(\partial \omega+d \omega-\frac{1}{2}[J \omega, \omega]\right) \equiv 0 \bmod I^{n+1} \Re \hat{\otimes} L$,

(d) $d^{2} \equiv 0 \bmod I^{n+1} \Re$.

Proof. We prove the lemma by induction on $n$. When $n=1$ the lemma is easily verified. Suppose that $n>1$ and that the lemma is true for $n-1$. By induction $d^{2} \equiv 0 \bmod I^{n} \Re$. Observe that

$$
d^{2} \omega \equiv \sum\left(d^{2} x_{i}\right) W_{i} \bmod I^{n+1} \Re \hat{\otimes} L
$$

Denote the universal enveloping algebra of $L$ by $U L$ and define the element $J \omega \wedge \omega$ of $\Re \hat{\otimes} U L$ by $J \omega \wedge \omega=\Sigma\left(J u_{i} \wedge u_{j}\right) U_{i} U_{j}$, where $\omega=\Sigma u_{i} U_{i}$. It is straightforward to verify that $\frac{1}{2}[J \omega, \omega]=J \omega \wedge \omega$ in $\Re \hat{\otimes} U L$ so that in $\Re \hat{\otimes} U L$ we have 


$$
\begin{aligned}
\partial\left(\partial \omega+d \omega-\frac{1}{2}[J \omega, \omega]\right)= & \partial(\partial \omega+d \omega-J \omega \wedge \omega) \\
= & d \partial \omega-J(\partial \omega) \wedge \omega+\omega \wedge \partial \omega \\
\equiv & d \partial \omega+J(d \omega-J \omega \wedge \omega) \wedge \omega \\
& -\omega \wedge(d \omega-J \omega \wedge \omega) \bmod I^{n+1} \Re \hat{\otimes} U L \\
\equiv & d(\partial \omega-J \omega \wedge \omega) \bmod I^{n+1} \Re \hat{\otimes} U L \\
\equiv & -d^{2} \omega \bmod d\left(I^{n} \Re\right) \hat{\otimes} U L \\
\equiv & -d^{2} \omega \bmod I^{n+1} \Re \hat{\otimes} U L .
\end{aligned}
$$

Therefore

$$
\partial\left(\partial \omega+d \omega-\frac{1}{2}[J \omega, \omega]\right) \equiv \sum\left(d^{2} x_{i}\right) W_{i} \bmod I^{n+1} \Re \hat{\otimes} L
$$

Since the $W_{i}$ represent linearly independent homology classes of $L$, and since the left-hand side of the previous equation is in $I^{n+1} \mathfrak{N} \hat{\otimes}(\partial L)$, it follows that $d^{2} x_{i} \in$ $I^{n+1} \Re$ so that $d^{2} \equiv 0 \bmod I^{n+1} \Re$ and

$$
\partial\left(\partial \omega+d \omega-\frac{1}{2}[J \omega, \omega]\right) \equiv 0 \bmod I^{n+1} \Re \hat{\otimes} L .
$$

To complete the proof of (3.7) we construct a sequence $\left(\omega_{n}\right)_{n \geqslant 1}$ of elements of $\prod_{p=0}^{\infty} \Re^{p+1} \otimes L_{p}$ and a sequence of derivations $\left(d_{n}\right)_{n \geqslant 1}$ of $\mathfrak{K}$, each of degree 1 , that satisfy:

(a) $\omega_{1}=\sum x_{i} W_{i}$ and $d_{i}=0$,

(b) $\partial \omega_{n}+d_{n} \omega_{n}-\frac{1}{2}\left[J \omega_{n}, \omega_{n}\right] \equiv 0 \bmod I^{n+1} \Re \hat{\otimes} L$,

(c) $\omega_{n+1} \equiv \omega_{n} \bmod I^{n+1} \Re \hat{\otimes} L$,

(d) $d_{n+1} x_{i} \equiv d_{n} x_{i} \bmod I^{n+1} \mathfrak{N}$.

Before giving the proof of the existence of $\left(\omega_{n}\right)$ and $\left(d_{n}\right)$ we need to introduce some notation. For each finite sequence $I=\left(i_{1}, i_{2}, \ldots, i_{p}\right)$ of elements of $S$, the set indexing the $x_{i}$ 's, denote by $x_{I}$ the element $x_{i_{1}} \wedge x_{i_{2}} \wedge \cdots \wedge x_{i_{p}}$ of $\Re$. For each positive integer $p$, choose a subset $\mathcal{G}(p)$ of $S \times S \times \cdots \times S$ ( $p$ times) such that $\left\{x_{I}\right.$ : $\in \mathcal{G}(p), p \geqslant 1\}$ is a basis of $I \mathscr{R}$. Set $\mathcal{G}=\cup_{p=1}^{\infty} \mathcal{G}(p)$.

Each element $\mu$ of $I \Re \hat{\otimes} L$ can be written uniquely in the form $\Sigma_{I \in \mathcal{G}} x_{I} U_{I}$ where $U_{I} \in L$. We shall denote by $\mu(n)$ the "truncation" $\sum_{p \leqslant n} \Sigma_{I \in \mathcal{G}(p)} x_{I} U_{I}$ of $\mu$.

Set $\omega_{1}=\sum x_{i} W_{i}$ and $d_{1}=0$. Conditions (a) through (d) are trivially satisfied. Suppose that $n \geqslant 2$ and that $\omega_{1}, \omega_{2}, \ldots, \omega_{n-1}$ and $d_{1}, d_{2}, \ldots, d_{n-1}$ have already been constructed satisfying conditions (a) through (d). To construct $\omega_{n}$ and $d_{n}$ we need to find, for each $I \in \mathcal{G}(n)$, rational numbers $\left(a_{i}^{I}\right)_{i \in S}$ and an element $W_{I}$ of $L$ such that if we set

$$
\omega_{n}=\omega_{n-1}+\sum_{I \in \mathcal{S}(n)} x_{I} W_{I}, \quad d_{n} x_{i}=d_{n-1} x_{i}+\sum_{I \in \mathcal{S}(n)} a_{i}^{I} x_{I}
$$

then

$$
\partial \omega_{n}+d_{n} \omega_{n}-\frac{1}{2}\left[J \omega_{n}, \omega_{n}\right] \equiv 0 \bmod I^{n+1} \Re \hat{\otimes} L
$$


Since

$$
\partial \omega_{n-1}+d_{n-1} \omega_{n-1}-\frac{1}{2}\left[J \omega_{n-1}, \omega_{n-1}\right] \equiv 0 \bmod I^{n} \Re \hat{\otimes} L
$$

and since $d_{n-1}\left(I^{2} \Re\right) \subseteq I^{n+1} \Re$, it follows from a short calculation that if $\left(a_{i}^{I}\right)_{i \in S}$ and $W_{I}$ satisfy the equation

(*) $\sum_{I \in \mathcal{G}(n)}\left(x_{I} \partial W_{I}+\sum_{i \in S} a_{i}^{I} x_{I} W_{i}\right)=-\left(\partial \omega_{n-1}+d_{n-1} \omega_{n-1}-\frac{1}{2}\left[J \omega_{n-1}, \omega_{n-1}\right]\right)(n)$,

then $\omega_{n}$ and $d_{n}$ satisfy conditions (a) through (d).

It follows from (3.8) that

$$
\partial\left(\partial \omega_{n-1}+d_{n-1} \omega_{n-1}-\frac{1}{2}\left[J \omega_{n-1}, \omega_{n-1}\right]\right)(n)=0 .
$$

Since the homology classes of the $W_{i}$ span $H_{*}(L)$, we can find numbers $a_{i}^{I}$ and elements $W_{I}$ of $L$ such that equation $(*)$ is satisfied. Thus $\omega_{n}$ and $d_{n}$ can be constructed.

Define $\omega$ and $d$ by insisting that

$$
\omega \equiv \omega_{n} \bmod I^{n+1} \Re \hat{\otimes} L \quad \text { and } d x_{i} \equiv d_{n} x_{i} \bmod I^{n+1} \Re
$$

4. The main theorem. In this section we state the main theorem of this paper, give examples of its applications and outline some topological applications of the theorem.

(4.1) THEOREM. (a) Let $\Re$ be a 1-connected minimal cochain algebra of finite type and $L$ a connected minimal cochain algebra of finite type. If $\omega$ is a twisting cochain in $\Re \hat{\otimes} L$, then $(\omega, L)$ is a connection on $\Re$ if and only if $(\Re, \omega)$ is a connection on $L$.

(b) The minimal chain Lie algebra associated to a 1-connected minimal cochain algebra of finite type by a connection is unique up to an isomorphism that preserves the pairings (3.4) and (3.5) defined by the connection form. Dually, the minimal cochain algebra associated to a connected minimal chain Lie algebra of finite type is unique up to an isomorphism that preserves the pairings (3.4) and (3.5) defined by the connection form.

In the case when the minimal chain Lie algebra $L$ is associated to the minimal cochain algebra $\Re$ by a connection (equivalently, $\Re$ is associated to $L$ via a connection) we shall say that there is a connection between $\mathfrak{K}$ and $L$.

(4.2) EXAMPLES. (a) Let $n$ be an odd integer, $n>1$. Let

$$
\Re=\Lambda(a: \operatorname{deg} a=n, d a=0) \text { and } L=\mathbf{L}(X: \operatorname{deg} X=n-1, \partial X=0) .
$$

A connection between $\Re$ and $L$ is given by the connection form $\omega=a X$. In this case $I \Re$ is spanned by $a$ and $L$ by $X$ and both pairings associated to $\omega$ are given by $\langle a, s X\rangle=1$.

(b) Let $n$ be an even integer, $n \geqslant 2$. For

$$
\begin{gathered}
\Re=\Lambda\left(a, b: \operatorname{deg} a=n, \operatorname{deg} b=2 n-1, d a=0, d b=a^{2}\right), \\
L=\mathbf{L}(X: \operatorname{deg} X=n-1, \partial X=0),
\end{gathered}
$$


a connection between $\mathfrak{R}$ and $L$ is given by the connection form $a X+\frac{1}{2} b[X, X]$. Here the pairing $I H^{*}(\mathfrak{T}) \otimes s Q L \rightarrow \mathbf{Q}$ is given by $\langle a, s X\rangle=1$ and the pairing $Q L \otimes s H_{*}(L)$ is given by $\langle a, X\rangle=1,\langle b, X\rangle=0,\langle a,[X, X]\rangle=0,\langle b,[X, X]\rangle=2$.

(c) Theorem (4.1) can be used to compute the homology of connected minimal chain Lie algebras. For example, we can easily compute the homology of the chain Lie algebra $\left(\mathbf{L}\left(X_{1}, X_{2}, \ldots, X_{n}\right), \partial\right)$ where $\operatorname{deg} X_{k}=2 k-1, \partial X_{1}=0$ and $\partial X_{k}$ $=\frac{1}{2} \sum_{1+j=k}\left[X_{i}, X_{j}\right]$. Denote this Lie algebra by $L$. Let

$$
\Re=\Lambda\left(a, b: \operatorname{deg} a=2, \operatorname{deg} b=2 n+1, d a=0, d b=a^{n+1}\right) .
$$

The element $\omega$ of $\Re \hat{\otimes} L$ given by

$$
\omega=\sum_{k=1}^{n} a^{k} X_{k}+\frac{1}{2} \sum_{k=1}^{n} a^{k-1} b \sum_{i+j=n+k}\left[X_{i}, X_{j}\right]
$$

is a twisting cochain in $\mathfrak{N} \hat{\otimes} L$. Since $\omega \equiv \sum_{k=1}^{n} a^{k} X_{k} \bmod \mathfrak{N} \hat{\otimes} I^{2} L$, it follows that $(\omega, L)$ is a connection on $\Re$. By Theorem $(4.1),(\Re, \omega)$ is a connection on $L$. Since $\omega \equiv a X_{1}+\frac{1}{2} b \sum_{i+j=n+1}\left[X_{i}, X_{j}\right] \bmod I^{2} \Re \hat{\otimes} L$, it follows that the homology classes $\left\{X_{1}\right\}$ and $\left\{\Sigma_{i+j=n+1}\left[X_{i}, X_{j}\right]\right\}$ are nontrivial in, and span $H_{*}(L)$.

(d) Often we want to find the chain Lie algebra associated to the minimal model $\Re$ of a 1-connected cochain algebra $Q$ with finite Betti numbers and compute the pairings (1.1) and (1.2) in low dimensions (a typical example being when $\mathbb{Q}$ is the de Rham complex of a 1-connected manifold with finite Betti numbers). This is most easily achieved by first finding a connection $(\omega, L)$ on $\mathcal{Q}$. If $(\tilde{\omega}, \tilde{L})$ is a connection on $\Re$, then it can be shown that $L \approx \tilde{L}$ (cf. [8, Proposition (6.2)]). The following example illustrates this and other techniques for dealing with not so trivial examples.

Let $Q$ be the truncated polynomial algebra

$$
\Lambda\left(c_{1}, c_{2}: \operatorname{deg} c_{1}=2, \operatorname{deg} c_{2}=4\right) / 9
$$

where $\mathcal{G}$ is the ideal of $\Lambda\left(c_{1}, c_{2}\right)$ generated by $c_{1}^{3}, c_{1} \wedge c_{2}$ and $c_{2}^{2}$. We will compute a connection on $Q$ and the pairing $Q \Re \otimes H_{*}(L) \rightarrow \mathbf{Q}$ in low dimensions, where $\Re$ is the minimal model of $\mathscr{Q}$ and where $L$ is the Lie algebra associated to $\mathscr{Q}$ by a connection.

A connection $(\omega, L)$ on $\mathcal{Q}$ is given by

$$
\begin{gathered}
L=\mathbf{L}\left(X_{1}, X_{11}, X_{2}: \operatorname{deg} X_{1}=1, \operatorname{deg} X_{11}=\operatorname{deg} X_{2}=3, \partial X_{2}=0,\right. \\
\omega=c_{1} X_{1}+c_{1}^{2} X_{11}+c_{2} X_{2} .
\end{gathered}
$$

Set $\mathscr{M}^{\prime}=\Lambda\left(x_{1}, x_{2}, u_{1,11}, u_{1,2}, u_{12,11}, u_{12,2}\right)$ where $\operatorname{deg} x_{1}=2, \operatorname{deg} x_{2}=4, \operatorname{deg} u_{1,11}$ $=\operatorname{deg} u_{1,2}=5, \operatorname{deg} u_{2,2}=7, \operatorname{deg} u_{12,11}=\operatorname{deg} u_{12,2}=8$ and where $d x_{1}=d x_{2}=0$, $d u_{1,11}=x_{1}^{3}, d u_{1,2}=x_{1} \wedge x_{2}, d u_{2,2}=x_{2}^{2}, d u_{12,11}=x_{1}^{2} \wedge u_{1,2}-x_{2} \wedge u_{1,11}, d u_{12,2}=$ $u_{1,2} \wedge x_{2}-x_{1} \wedge u_{2,2}$. Define a d.g. algebra map $\rho: \mathfrak{T}^{\prime} \rightarrow \mathbb{Q}$ by $\rho\left(x_{1}\right)=c_{1}, \rho\left(x_{2}\right)=$ $c_{2}$ and $\rho\left(u_{I, J}\right)=0$ for all $I, J$. It is easy to check that $\rho$ induces an isomorphism on cohomology in degrees $\leqslant 9$. Consequently, there is an inclusion of $\mathfrak{K}^{\prime}$ into the minimal model $\Re$ of $Q$ that is an isomorphism in degrees $\leqslant 8$. 
The method we use to compute the pairing $Q \Re \otimes H_{*}(L) \rightarrow \mathbf{Q}$ in degrees $\leqslant 8$ is to find an element $\omega^{\prime}$ of $\Re^{\prime} \otimes L$ that is an "approximation" to a connection form in $\Re \hat{\otimes} L$. Specifically, we will find an element $\omega^{\prime}$ of $\Re \hat{\otimes} L$ such that there exists a connection form $\tilde{\omega}$ in $\Re \hat{\otimes} L$ such that

$$
\tilde{\omega} \equiv \omega^{\prime} \bmod \bigoplus_{p \geqslant 9}\left(\Re^{p} \otimes L_{p-1}\right) .
$$

The procedure for doing this is to find a sequence $\left(\omega_{r}^{\prime}\right)_{r \geqslant 1}$ of elements of $\Re \hat{\otimes} L$ such that:

(a) $\omega_{1}^{\prime}=x_{1} X_{1}+x_{1}^{2} X_{11}+x_{2} X_{2}$,

(b) $\partial \omega_{r}^{\prime}+d \omega_{r}^{\prime}-\frac{1}{2}\left[J \omega_{r}^{\prime}, \omega_{r}^{\prime}\right] \equiv 0 \bmod \Re \hat{\otimes} I^{r+1} L$,

(c) $\omega_{r+1}^{\prime} \equiv \omega_{r}^{\prime} \bmod I^{r+1} \mathfrak{N} \hat{\otimes} L$.

To find $\omega_{r+1}^{\prime}$ given $\omega_{r}^{\prime}$, set $\omega_{r+1}^{\prime}=\omega_{r}^{\prime}+\mu$ where $\mu$ is an element of $\Re \hat{\otimes} I^{r+1} L$ which satisfies the equation

$$
-d \mu \equiv \partial \omega_{r}^{\prime}+d \omega_{r}^{\prime}-\frac{1}{2}\left[J \omega_{r}^{\prime}, \omega_{r}^{\prime}\right] \bmod \Re \hat{\otimes} I^{r+2} L
$$

We begin with $\omega_{1}^{\prime}=x_{1} X_{1}+x_{1}^{2} X_{11}+x_{2} X_{2}$. Now

$$
\begin{aligned}
& \partial \omega_{1}^{\prime}+d \omega_{1}^{\prime}-\frac{1}{2}\left[J \omega_{1}^{\prime}, \omega_{1}^{\prime}\right]=-\frac{1}{2} x_{1}^{4}\left[X_{11}, X_{11}\right]-\frac{1}{2} x_{2}^{2}\left[X_{2}, X_{2}\right]-x_{1}^{3}\left[X_{1}, X_{11}\right] \\
&-x_{1} \wedge x_{2}\left[X_{1}, X_{2}\right]-x_{1}^{2} \wedge x_{2}\left[X_{11}, X_{2}\right] \\
&=-d\left(\frac{1}{2} x_{1} \wedge u_{1,11}\left[X_{11}, X_{11}\right]+\frac{1}{2} u_{2,2}\left[X_{2}, X_{2}\right]\right. \\
&\left.+u_{1,11}\left[X_{1}, X_{11}\right]+u_{1,2}\left[X_{1}, X_{2}\right]+x_{1} \wedge u_{1,2}\left[X_{11}, X_{2}\right]\right) .
\end{aligned}
$$

Thus

$$
\begin{aligned}
\omega_{2}^{\prime}= & \omega_{1}^{\prime}+\frac{1}{2} x_{1} \wedge u_{1,11}\left[X_{11}, X_{11}\right]+\frac{1}{2} u_{2,2}\left[X_{2}, X_{2}\right] \\
& +u_{1,11}\left[X_{1}, X_{11}\right]+u_{1,2}\left[X_{1}, X_{2}\right]+x_{1} \wedge u_{1,2}\left[X_{11}, X_{2}\right] .
\end{aligned}
$$

After a similar, but more tedious calculation, one can show that

$$
\begin{aligned}
\omega_{3}^{\prime}= & \omega_{2}^{\prime}+u_{12,2}\left[\left[X_{1}, X_{2}\right] X_{2}\right]+u_{12,11}\left[X_{2}\left[X_{1}, X_{11}\right]\right] \\
& +x_{1} \wedge u_{12,2}\left[\left[X_{11}, X_{2}\right] X_{2}\right]+x_{1} \wedge u_{12,11}\left[\left[X_{11}, X_{2}\right] X_{11}\right] .
\end{aligned}
$$

If we continued the procedure above and found a sequence $\left(\omega_{r}^{\prime}\right)$ satisfying (a), (b) and (c) above, then we could define a connection form $\tilde{\omega}$ in $\Re \hat{\otimes} L$ by insisting that $\tilde{\omega} \equiv \omega_{r}^{\prime} \bmod \Re \hat{\otimes} I^{r+1} L$.

Set $\omega^{\prime}=\omega_{3}^{\prime}$. A short computation shows that

$$
\partial \omega^{\prime}+d \omega^{\prime}-\frac{1}{2}\left[J \omega^{\prime}, \omega^{\prime}\right] \equiv 0 \bmod \bigoplus_{p \geqslant 9}\left(\Re^{p+1} \otimes L_{p-1}\right) .
$$

Thus we have found our approximation to a connection $\tilde{\omega}$ on $\Re$. Observe that

$$
\begin{aligned}
\omega^{\prime} \equiv & x_{1} X_{1}+x_{2} X_{2}+\frac{1}{2} u_{2,2}\left[X_{2}, X_{2}\right]+u_{1,11}\left[X_{1}, X_{11}\right]+u_{1,2}\left[X_{1}, X_{2}\right] \\
& +u_{12,2}\left[\left[X_{1}, X_{2}\right] X_{2}\right]+u_{12,11}\left[X_{2}\left[X_{1}, X_{11}\right]\right] \bmod I^{2} \Re \hat{\otimes} L .
\end{aligned}
$$


The following table gives dual bases of $Q \mathfrak{N}$ and $H_{*}(L)$ in low dimensions:
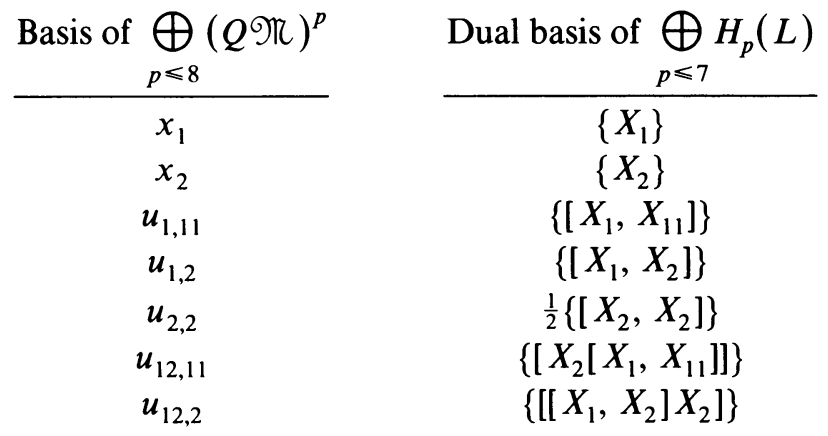

As remarked in the introduction, if $K$ is a 1-connected space with finite Betti numbers, if $\Re_{K}$ is Sullivan's minimal model of $K$ and if $L_{K}$ is the Lie algebra model of $K$, then $\mathfrak{R}_{K}$ and $L_{K}$ correspond under the duality theory, $H^{*}\left(\mathfrak{N}_{K}\right) \approx H^{*}(K ; \mathbf{Q})$, $Q \Re \approx \operatorname{Hom}_{\mathbf{Z}}\left(\pi_{*}(K), \mathbf{Q}\right), H_{*}(L) \approx s^{-1} \pi_{*}(K) \otimes_{\mathrm{z}} \mathbf{Q}$ and $Q L \approx s^{-1} \tilde{H}_{*}(K ; \mathbf{Q})$. In (3.3), Example (a) corresponds to $S^{2}$, and (b) to $S^{3} \times S^{3}$. In (4.2), Examples (a) and (b) correspond to $S^{n}$ where $n$ is odd and $n$ is even, respectively. Example (c) corresponds to $C P^{n}$ and (d) to the 4-skeleton of $B U$.

If $K$ is a 1-connected rational space with finite Betti numbers, then $\Re_{K}$ determines the Postnikov tower of $K[6,15]$ and $L_{K}$ a homology decomposition of $K$ $[13,18]$. The topological implication of Theorems (3.7) and (4.1) is that we can inductively compute the $k$-invariants of $K$ from the characteristic maps of a homology decomposition of $K$ and vice versa (see [8 or 10] for example).

5. A proof of Theorem (4.1). The proof of (4.1) is a composite of results of Quillen [14, Appendix B], Sullivan [16], Lehmann [9] and Baues and Lemaire [2]. We begin

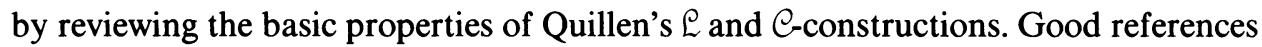
for this material include [2] and [13] as well as Appendix B of Quillen's paper [14].

A chain coalgebra is a d.g. coalgebra $C$ with a differential $\partial$ of degree -1 and a coaugmentation $\eta: \mathbf{Q} \rightarrow C$. We shall denote the cokernel of $\eta$ by $\bar{C}$ and the image in $\bar{C}$ of an element $c$ of $C$ by $\bar{c}$. The set of primitive elements $\{c \in C: \Delta c=c \otimes 1+1$ $\otimes c\}$ of $C$ will be denoted by $P C$. We say that $C$ is $r$-connected if the graded vector space $\bar{C}$ is $r$-connected. A chain coalgebra $C$ is commutative if

$$
\Delta c=\sum a_{i} \otimes b_{i}=\sum(-1)^{\operatorname{deg} a_{i} \operatorname{deg} b_{i}} b_{i} \otimes a_{i}
$$

for all $c \in C$.

Let $C$ be a commutative chain coalgebra. The $\mathcal{L}$-construction on $C$ is a chain Lie algebra $\mathcal{L}(C)$ that, as a Lie algebra, is isomorphic to $\mathbf{L}\left(s^{-1} \bar{C}\right)$. As a d.g. vector space, $Q \mathcal{L}(C) \approx s^{-1} \bar{C}$.

Let $L$ be a chain Lie algebra. The $C$-construction on $L$ is a cocommutative chain coalgebra $C(L)$ that, as a coalgebra, is isomorphic to the symmetric coalgebra $S(s L)$ on $s L$. As a d.g. vector space, $P \bigodot(L)$ is isomorphic to $s L$.

There is a canonical inclusion $i: s^{-1} \bar{C} \rightarrow \varrho(C)$ that corresponds to the canonical inclusion $s^{-1} \bar{C} \rightarrow \mathbf{L}\left(s^{-1} \bar{C}\right)$. There is a canonical projection $p: \mathcal{C}(L) \rightarrow s L$ that 
corresponds to the canonical projection of $S(s L)$ onto $s L$. In general, neither $i$ nor $p$ is a d.g. vector space map.

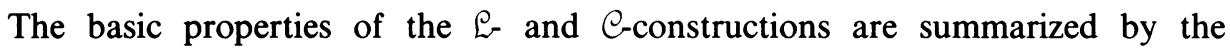
following theorem.

(5.1) THEOREM. Let $C$ be a cocommutative chain coalgebra and L a chain Lie algebra. There is a natural one-to-one correspondence between d.g. coalgebra maps $C \rightarrow \mathcal{C}(L)$ and d.g. Lie algebra maps $\mathcal{L}(C) \rightarrow L:$ the map $\varphi: C \rightarrow \mathcal{C}(L)$ corresponds to the map $\psi: \mathcal{L}(C) \rightarrow L$ if and only if the linear maps $\psi \circ i \circ s^{-1}: \bar{C} \rightarrow L$ and $s^{-1} \circ p \circ \varphi: \bar{C} \rightarrow L$ are equal. Moreover, if $H_{*}(C)$ is 1-connected and $H_{*}(L)$ is connected, and if the maps $\varphi: C \rightarrow \mathcal{C}(L)$ and $\psi: \mathcal{L}(C) \rightarrow L$ correspond as above, then $\varphi$ induces an isomorphism on homology if and only if $\psi$ induces an isomorphism on homology.

Let $Q$ be a cochain algebra of finite type and $L$ a chain Lie algebra. Denote the dual coalgebra $\operatorname{Hom}(\mathscr{Q}, \mathbf{Q})$ of $\mathbb{Q}$ by $\mathbb{Q}^{*}$. Each twisting cochain $\omega$ in $\mathbb{Q} \hat{\otimes} L$ determines a chain Lie algebra map $\theta: \mathcal{L}\left(\mathbb{Q}^{*}\right) \rightarrow L$ that we shall call the holonomy map associated to $\omega$. If $\omega=\sum u_{i} U_{i}$ and $\varphi \in \mathbb{Q}^{*}$, define $\langle\omega, \bar{\varphi}\rangle=\Sigma \varphi\left(u_{i}\right) U_{i}$. Observe that this is a finite sum so that $\langle\omega, \bar{\varphi}\rangle \in L$. The holonomy map $\theta: \mathcal{L}\left(\mathbb{Q}^{*}\right) \rightarrow L$ is then defined by

$$
\theta\left(s^{-1} \bar{\varphi}\right)=\langle\omega, \bar{\varphi}\rangle \text { for all } \varphi \in \mathbb{Q}^{*} .
$$

Using the fact that $\omega$ is a twisting cochain, one can show that $\theta$ is a d.g. Lie algebra map (see [14, Appendix B]).

We would like to characterize the connections on $Q$ as the pairs $(\omega, L)$ where the associated holonomy map $\theta: \mathcal{L}\left(\mathbb{Q}^{*}\right) \rightarrow L$ induces an isomorphism on homology. To do this we need the following lemma. A proof of it can be found in [2, p. 226].

(5.2) Lemma. Let $L_{1}$ and $L_{2}$ be free chain Lie algebras with connected homology. A d.g. Lie algebra map $\varphi: L_{1} \rightarrow L_{2}$ induces an isomorphism on homology if and only if $Q \varphi: Q L_{1} \rightarrow Q L_{1}$ induces an isomorphism on homology.

We can now prove the desired characterization of connections on a cochain algebra.

(5.3) LEMMA. Let $Q$ be a 1-connected cochain algebra of finite type and L a connected minimal chain Lie algebra. If $\omega$ is a twisting cochain in $Q \hat{\otimes} L$, then $(\omega, L)$ is a connection on $\mathbb{Q}$ if and only if the associated holonomy map $\theta: \mathcal{L}\left(\mathbb{Q}^{*}\right) \rightarrow L$ induces an isomorphism on homology.

Proof. Let $\left(X_{i}\right)$ be a set that freely generates $L$. Suppose that $\omega \equiv \sum w_{i} X_{i}$ $\bmod Q \hat{\otimes} I^{2} L$. Since $\partial$ (the differential in $L$ ) is decomposable, the twisting cochain condition implies that $d \omega \equiv 0 \bmod Q \hat{\otimes} I^{2} L$. That is, each $w_{i}$ is closed in $\mathcal{Q}$.

The holonomy map $\theta: \mathcal{L}\left(\mathbb{Q}^{*}\right) \rightarrow L$ induces the map $s^{-1} \bar{\varphi} \mapsto \Sigma \varphi\left(w_{i}\right) X_{i}$ on indecomposables $\left(\varphi \in \mathbb{Q}^{*}\right)$. Observe that $(\omega, L)$ is a connection on $\mathbb{Q}$ if and only if this map induces an isomorphism on homology. The result now follows from (5.2). 
To prove the analogous result for connections on Lie algebras, we need an analogue of (5.2) for free commutative cochain algebras. The following proposition is proved in Lehmann [9, Proposition V.7] and is implicit in Theorem (2.2) of [16].

(5.4) Proposition. Let $\mathbb{Q}$ be a free, connected cochain algebra with 1-connected homology. Let $\mathfrak{N}$ be a minimal cochain algebra. Ad.g. algebra map $\rho: \mathfrak{R} \rightarrow \mathbb{Q}$ induces an isomorphism on homology if and only if $Q \rho: Q \Re \rightarrow Q Q$ induces an isomorphism on homology.

An easy consequence of the above proposition is the natural analogue of (5.2) for free commutative cochain algebras.

(5.5) LemMA. Let $\mathbb{Q}$ and $\mathbb{Q}^{\prime}$ be connected, free cochain algebras with 1-connected homology. A d.g. algebra map $\varphi: \mathbb{Q} \rightarrow \mathbb{Q}^{\prime}$ induces an isomorphism on homology if and only if $Q \varphi: Q Q \rightarrow Q A^{\prime}$ induces an isomorphism on homology.

Let $\mathcal{Q}$ be a cochain algebra of finite type and $L$ a chain Lie algebra. For a twisting cochain $\omega \in \mathbb{Q} \hat{\otimes} L$, we shall denote by $\theta^{t}: \mathbb{Q}^{*} \rightarrow \mathcal{C}(L)$ the d.g. coalgebra map that corresponds to the holonomy map $\theta: \mathcal{L}\left(\mathbb{Q}^{*}\right) \rightarrow L$ associated to $\omega$.

We can now characterize connections on chain Lie algebras in terms of their holonomy maps.

(5.6) LEMMA. Let L be a connected chain Lie algebra of finite type and let $\Re$ be a 1-connected minimal cochain algebra of finite type. If $\omega$ is a twisting cochain in $\Re \hat{\otimes} L$, then $(\Re, \omega)$ is a connection on $L$ if and only if the associated holonomy map $\theta: \mathcal{L}\left(\mathfrak{T}^{*}\right) \rightarrow L$ induces an isomorphism on homology.

Proof. Let $\left(x_{i}\right)$ be a set that freely generates $\mathfrak{T}$. Suppose that $\omega \equiv \sum x_{i} W_{i}$ $\bmod I^{2} \Re \hat{\otimes} L$. Since $d$ (the differential in $\Re$ ) is decomposable, the twisting cochain condition implies that $\partial \omega \equiv 0 \bmod I^{2} \mathfrak{R} \hat{\otimes} L$. That is, each $W_{i}$ is closed in $L$.

The holonomy map $\theta: \mathcal{L}\left(\Re^{*}\right) \rightarrow L$ associated to $\omega$ induces an isomorphism on homology if and only if the coalgebra map $\theta^{t}$ : $\Re^{*} \rightarrow \mathcal{C}(L)$ induces an isomorphism on homology. Since $\Re$ is of finite type, $\operatorname{Hom}\left(\Re^{*}, \mathbf{Q}\right) \approx \Re$, and since $L$ is connected and of finite type, $\operatorname{Hom}(C(L), \mathbf{Q})$ is a 1-connected, free cochain algebra. Thus $\theta^{t}$ induces an isomorphism on homology if and only if its dual $\boldsymbol{\theta}^{t^{*}}$ : $\operatorname{Hom}(\mathcal{C}(L), \mathbf{Q}) \rightarrow \mathfrak{T}$ induces an isomorphism on homology.

Observe that there is a natural isomorphism $Q \operatorname{Hom}(C(L), \mathbf{Q}) \approx \operatorname{Hom}(s L, \mathbf{Q})$ and that, with respect to this isomorphism, $Q \theta^{t^{*}}$ takes the functional $\psi$ on $s L$ to $\sum x_{i} \psi\left(s W_{i}\right)$ (a finite sum). This follows from (5.1). The result now follows from (5.5).

The first assertion of (4.1) now follows directly from (5.3) and (5.6).

Let $L$ be a connected chain Lie algebra of finite type. Suppose that $\left(\Re_{1}, \omega_{1}\right)$ and $\left(\Re_{2}, \omega_{2}\right)$ are connections on $L$ with associated holonomy maps $\theta_{j}: \mathcal{L}\left(\Re_{j}^{*}\right) \rightarrow L$ $(j=1,2)$. As in the proof of $(5.6)$, the dual of the adjoint of $\boldsymbol{\theta}_{j}$,

$$
\boldsymbol{\theta}_{j}^{t^{*}}: \operatorname{Hom}(\mathcal{C}(L), \mathbf{Q}) \rightarrow \mathfrak{K}_{j}, \quad j=1,2,
$$


induces an isomorphism on homology. In fact, $\theta_{j}^{t^{*}}$ splits as the following argument shows. Let $\rho: \mathfrak{T} \rightarrow \operatorname{Hom}(\mathcal{C}(L), \mathbf{Q})$ be a minimal model of $\operatorname{Hom}(\mathcal{C}(L), \mathbf{Q})$. (For a definition of minimal model, see $[6,15]$.) The composite $\theta_{j}^{t^{*}} \circ \rho: \Re_{\rightarrow} \Re_{j}$ induces an isomorphism on homology. Because $\Re$ and $\mathfrak{R}_{j}$ are 1-connected and minimal, $\theta_{j}^{t^{*}} \circ \rho$ is an isomorphism. Define

$$
\Psi_{j}: \Re_{j} \rightarrow \operatorname{Hom}(\varrho(L), \mathbf{Q})
$$

by $\Psi_{j}=\rho \circ\left(\theta_{j}^{t^{*}} \circ \rho\right)^{-1}$. Observe that $\theta_{j}^{t^{*}} \circ \Psi_{j}$ equals id: $\Re_{j} \rightarrow \Re_{j}$.

Define a d.g. algebra map $\Phi: \Re_{1} \rightarrow \Re_{2}$ by $\Phi=\theta_{2}^{t^{*}} \circ \Psi_{1}$. Since $\Phi$ induces an isomorphism on homology and since both $\mathfrak{K}_{1}$ and $\mathfrak{K}_{2}$ are 1-connected and minimal, $\Phi$ is an isomorphism.

To see that the diagram

$$
\begin{array}{lll}
Q \Re_{1} \otimes s H_{*}(L) & & \\
Q \Phi \otimes \mathrm{id} \downarrow & \searrow & \mathbf{Q} \\
Q \Re_{2} \otimes s H_{*}(L) & &
\end{array}
$$

commutes, observe that the diagram

$$
\begin{aligned}
& H_{*}(Q \operatorname{Hom}(\mathcal{C}(L), \mathbf{Q})) \stackrel{\left(Q \theta_{1}^{r^{*}}\right) *}{\rightarrow} Q \Re_{1} \\
& \left(Q \theta_{2}^{t^{*}}\right)_{*} \searrow \quad \downarrow Q \Phi \\
& Q \Re_{2}
\end{aligned}
$$

commutes and that with respect to the identification

$$
\operatorname{Hom}\left(s H_{*}(L), \mathbf{Q}\right) \approx H_{*}(Q \operatorname{Hom}(\mathcal{C}(L), \mathbf{Q})),
$$

the map $\theta_{j}^{t^{*}}$ is the polarity of the pairing $Q \mathfrak{T}_{j} \otimes s H_{*}(L) \rightarrow \mathbf{Q}$ given by $\omega_{j}$.

If, in addition, $L$ is minimal, then it follows from (4.1)(a) that the homology of $\operatorname{Hom}(\mathcal{C}(L), \mathbf{Q})$ is isomorphic to $\operatorname{Hom}(s Q L, \mathbf{Q})$. Using this and the fact that the diagram

$$
\begin{array}{rr}
H^{*}(\operatorname{Hom}(\mathcal{C}(L), \mathbf{Q})) \stackrel{\theta_{1}^{r^{*}}}{\rightarrow} H^{*}\left(\Re_{1}\right) \\
\theta_{2}^{r^{*} \searrow} & \begin{array}{r}
\downarrow \Phi^{*} \\
\end{array} \\
H^{*}\left(\Re_{2}\right)
\end{array}
$$

commutes, it follows that the diagram

$$
\begin{aligned}
& I H^{*}\left(\Re_{1}\right) \otimes s Q L \\
& I \Phi^{*} \otimes \mathrm{id} \downarrow \\
& \text { Q } \\
& I H^{*}\left(\mathfrak{T}_{2}\right) \otimes s Q L
\end{aligned}
$$

commutes up to homotopy. 
The homotopy class of $\Phi$ is uniquely determined, for if

$$
\begin{aligned}
\operatorname{Hom}(\mathcal{C}(L), \mathbf{Q}) \stackrel{\theta_{1}^{\theta^{*}}}{\rightarrow} & \Re_{1} \\
\theta_{2}^{t^{*}} \searrow & \downarrow \Phi^{\prime} \\
& \Re_{2}
\end{aligned}
$$

commutes up to homotopy, then $\Phi^{\prime}=\Phi^{\prime} \circ \theta_{1}^{t^{*}} \circ \Psi_{1} \simeq \theta_{2}^{t^{*}} \circ \Psi_{1}=\Phi$.

The uniqueness of the Lie algebra in the dual case is proved by a similar argument.

(5.7) CoRollary. If $L$ and $\Re$ correspond under the duality theory, then $L$ is isomorphic to the minimal model of $\mathcal{L}\left(\mathfrak{T}^{*}\right)$ and $\mathfrak{T}$ is isomorphic to the minimal model of $\operatorname{Hom}(\mathcal{C}(L), \mathbf{Q})$.

It follows from Theorem 3.1.1 in [5] in the real case, and Theorem (6.9) in [18] in the rational case, that the universal enveloping algebra of Chen's Lie algebra model of a space is isomorphic to the Adams-Hilton model of the space (defined in [2]). This fact, together with (5.7) provides a correct proof of Proposition (3.6) in [2].

\section{REFERENCES}

1. C. Allday, Rational Whitehead product and a spectral sequence of Quillen. II, Houston J. Math. 3 (1977), 301-308.

2. H. J. Baues and J. M. Lemaire, Minimal models in homotopy theory, Math. Ann. 225 (1977), $219-242$.

3. E. H. Brown, Jr., Twisted tensor products. I, Ann. of Math. (2) 69 (1959), 223-246.

4. K.-T. Chen, Extension of $C^{\infty}$ function algebra by integrals and Malcev completion of $\pi_{1}$, Adv. in Math. 23 (1977), 181-210.

5. __ Iterated path integrals, Bull. Amer. Math. Soc. 83 (1977), 831-879.

6. P. Deligne, P. Griffiths, J. Morgan and D. Sullivan, Real homotopy theory of Kähler manifolds, Invent. Math. 29 (1975), 245-274.

7. V. K. A. M. Gugenheim, On a perturbation theory for the homology of the loop-space, preprint.

8. R. M. Hain, Iterated integrals, minimal models and rational homotopy theory, $\mathrm{Ph}$.D. thesis, Univ. of Illinois, 1980.

9. D. Lehmann, Théorie homotopique des formes différentielles, Astérisque 45 (1977).

10. J. M. Lemaire, Algèbres connexes it homologie de espace de lacets, Lecture Notes in Math., vol. 422, Springer-Verlag, Berlin, Heidelburg and New York, 1974.

11. __ Modèles minimaux pour les algèbres de chaines, Publ. Dép. Math. (Lyon) (3) 13 (1976), $13-26$.

12. J. Milnor and J. C. Moore, On the structure of Hopf algebras, Ann. of Math. (2) 81 (1965), 211-264.

13. J. Neisendorfer, Lie algebras, coalgebras and rational homotopy theory for nilpotent spaces, Pacific J. Math. 74 (1978), 429-460.

14. D. Quillen, Rational homotopy theory, Ann. of Math. (2) 90 (1969), 205-295.

15. D. Sullivan, Topology of manifolds and differential forms, Manifolds-Tokyo 1973, edited by Akio Hattori, Univ. of Tokyo Press, Tokyo, 1975.

16. , Infinitesimal computations in topology, Inst. Hautes Études Sci. Publ. Math. 47 (1977), 269-331.

17. Y. Felix, Modèles bifiltrés: une plaque tournante en homotopie rationnelle, Canad. J. Math. 33 (1981), 1448-1458.

18. R. M. Hain, Iterated integrals and homotopy periods, Mem. Amer. Math. Soc. (to appear)

19. D. Tanré, Modèles de Chen, Quillen, Sullivan, Publ. U.E.R. Math. Pures Appl. IRMA 2 (1980), No. 1, Exp. No. 2, 8 pp.

20. __ Dualite d'Eckmann-Hilton à travers les modèles de Chen-Quillen-Sullivan, Cahiers Topologie Géom. Différentielle 22 (1981), 53-59.

Department of Mathematics, University of Washington, Seattle, Washington 98195 